\title{
Study on drug utilization pattern in conservative management of patients with pancreatitis in a tertiary care teaching hospital
}

\author{
Mohanraj P. ${ }^{1 *}$, Abinaya S. ${ }^{1}$, Shalini K. ${ }^{1}$, Gopalakrishnan G. ${ }^{1}$, Ravichandran K. ${ }^{2}$
}

\begin{abstract}
${ }^{1}$ Department of Pharmacy, Annamalai University, Annamalai Nagar, Tamil Nadu, India
${ }^{2}$ Department of Surgery, Rajah Muthiah Medical College and Hospital, Annamalai University, Annamalai Nagar, Tamil Nadu, India
\end{abstract}

Received: 12 September 2021

Accepted: 04 October 2021

\section{*Correspondence:}

Dr. Mohanraj P.,

Email: pmohanraj1998@gmail.com

Copyright: (c) the author(s), publisher and licensee Medip Academy. This is an open-access article distributed under the terms of the Creative Commons Attribution Non-Commercial License, which permits unrestricted non-commercial use, distribution, and reproduction in any medium, provided the original work is properly cited.

\section{ABSTRACT}

Background: Drug utilization plays a key role in helping the healthcare system to understand, interpret and improve the prescribing, administration and use of medications. The objective of the study was to assess the drug utilization pattern of drugs prescribed in conservative management of pancreatitis patients in a tertiary care teaching hospital.

Methods: This prospective observational study was conducted in department of surgery, Rajah Muthiah Medical College and Hospital, Annamalai University. The study period was six months from November 2019 to April 2020. A total of 115 patients were selected based on inclusion and exclusion criteria.

Results: The study population containing of $90 \%(n=104)$ males and $10 \%(n=11)$ females were included in the study. Most of the patients between age group of 25-44 years (48\%). The most common etiology was alcoholic $68 \%(\mathrm{n}=78)$. $7 \%$ of population with comorbidities (5\% hypertension, $2 \%$ diabetes mellitus). In analgesics, tramadol (28\%) and in antibiotics cefotaxime $21 \%$ was most prescribed. Lactated ringer solution $(36 \%)$ was prescribed most as intravenous hydration. $7 \%(n=8)$ and $3 \%(n=4)$ of patients receiving enteral nutrition and parenteral nutrition. The average number of drugs per prescription was 8.5 . Out of 1205 drugs, $61 \%$ of drugs prescribed in generic form, $39 \%$ were prescribed in brand name. The study analyzed that $69 \%$ of drugs were prescribed from essential drugs list (EDL) 2019 and $84 \%$ drugs prescribed from national list of essential medicines (NLEM) 2015. Number of prescriptions with injection were 79.07\%. The overall antibiotic encounter rate $15 \%$. Prescribed daily dose/defined daily dose (PDD/DDD) ratio of folic acid was 5.

Conclusions: Drug utilization study can help in evaluating the quality of care given to the pancreatitis patients and promote rational use of medicines.

Keywords: Drug utilization, Defined daily dose, Prescribed daily dose, Prescribing pattern, Pancreatitis

\section{INTRODUCTION}

Drug utilization research is an invaluable tool for all involved in drug and health policies and related decisionmaking. Drug utilization is defined by World Health Organization (WHO) in 1977 as "marketing, distribution, prescription and use of drugs in a society, with special emphasis on the resulting medical, social and economic consequences". ${ }^{1}$ Research on drug utilization focus on factors related to prescribing, dispensing, administering and taking of medication and its associated events.
The ultimate purpose of drug utilization research is to contribute the optimal quality of drug therapy by Rational use of drug requires that patient receive medication appropriate to their clinical needs, in dose that meet their own individual requirements for the adequate period and the lowest cost to them and their community, identifying, documenting, analyzing problems in drug utilization and monitoring the consequences. The major factors can be patients, prescribers, work place and supply system. Therefore, it is important to recognize negative consequences of inappropriate drug use to promote 
rational use of drug. ${ }^{2}$ Irrational drug use and polypharmacy are the most common problem in clinical scenario. There is a scope for continuous research to identify more effective and safer drug utilization pattern among pancreatitis patients.

Hence this study was designed to evaluate the drug utilization pattern among pancreatitis patients in a tertiary care teaching hospital.

\section{METHODS}

It was a prospective observational study. The study will be conducted in department of surgery in Rajah Muthiah Medical College and Hospital, Annamalai University, Chidambaram, Tamil Nadu, a 1250 bed multi-speciality, tertiary care teaching hospital located in South India. The period of the study was 6 months (from November 2019 to April 2020).

\section{Inclusion criteria}

Patients of both genders with age group of 18 to 75 years and who are admitted in the department of surgery were included in the study.

\section{Exclusion criteria}

Patients unable to communicate i.e. patients on ventilation or critically ill (coma) patients requiring intensive care unit (ICU) admission; patients who are unwilling to participate; pregnant and lactating women; and patient with malignant pancreatitis were excluded from the study.

\section{Sample size}

A total of 115 patients in both genders with age group from 18-75 year were included in the study.

Sample size was calculated by using the formula as below.

$\mathrm{N}=\frac{z^{2} p(1-p)}{d^{2}}$

\section{Data analysis}

We collected the following data: demographic data of patients, prescription details like date, number of drugs, names of individual drugs (brand/generic), any fixed-dose combination prescribed, Intravenous fluids, whether the prescribed drugs were available from the hospital pharmacy or to be a bought by the patient from a private chemist, dose, dosage form, dosing schedule, duration of treatment.

\section{Analysis of drug}

Data were analysed for who prescribing Indicators like average number of drugs per prescription, percentage of drugs prescribed in generic name, number of drugs prescribed from national list of essential medicines (NLEM) 2015, number of drugs prescribed from essential drugs list (EDL) 2019 and percentage of prescriptions with injections. ${ }^{3}$

The prescribed drugs were classified according to the anatomical therapeutic chemical (ATC) classification and defined daily dose (DDD), prescribed daily dose (PDD), and PDD/DDD ratio was calculated. ${ }^{4}$

The DDD of these drugs was calculated according to the WHO ATC/DDD system based on DDD's/1000 inhabitants per day.

\section{Utilization in $D D D$

$$
\begin{aligned}
& =\text { No.of packages used } \\
& \times \text { No. of DDD in a package }
\end{aligned}
$$

$$
\begin{aligned}
& \frac{\frac{D D D s}{1000} \text { inhabitants }}{\text { day }} \\
& =\frac{\text { Utilization in } D D D s}{\text { No. of inhabitants }} \times 1000 \\
& \times(\text { no.of days in the period of data collection })
\end{aligned}
$$

To overcome the inherent limitations of the DDD, the PDD has been used alongside the DDD. PDD gives the average daily amount of the drug which is actually prescribed. ${ }^{5}$

$$
P D D=\frac{\text { Total amount of drug }(\mathrm{mg})}{\text { Duration of hospital stay }}
$$

\section{Ethical consideration}

The study was conducted after obtaining permission from the institutional ethics committee (IEC). All the data collected as a part of this study was kept strictly confidential and used for the purpose of this study only.

\section{Statistical analysis}

Data was entered and analysed with Microsoft excel 2016. We used descriptive statistics to analyze the results. Percentage and averages of the variable were also calculated to compare the data with other findings.

\section{RESULTS}

Out of 115 patients receiving conservative management for pancreatitis during the study period, most of the patients are males; $90 \%(n=104)$ and $10 \%(n=11)$ were females. The mean age was 38.2 years, and the range was 20-75 years.

We observed that the most common etiology of pancreatitis was seen in alcoholic patients $68 \%(n=78)$ followed by gallstones $15 \%(\mathrm{n}=17)$, hyperlipidemic $8 \%$ $(\mathrm{n}=10)$, idiopathic $7 \%(\mathrm{n}=8)$ then drug induced $2 \%(\mathrm{n}=2)$. 
Overall etiology wise distribution of patients according to age are summarized in Table 1.

In our study most of the patients with risk factors of alcoholic consumption $(\mathrm{n}=90)$ followed by smoking $(n=30)$, both $(n=70)$ and obesity $(n=5) .11 \%(n=13)$ of patients are literate, other $89 \%(\mathrm{n}=102)$ are Illiterate in our study. $92 \%(n=106)$ living with family, only $8 \%(n=9)$ of patients are alone in our study.

In our study two comorbidities were found. They are hypertension $5 \%(n=6)$ and diabetes mellitus $2.5 \%(n=2)$.

Antiulcer agents $21 \% \quad(n=153)$ are most frequently prescribed drugs among patients, followed by analgesics $18 \% \quad(n=132)$, antibiotics $18 \% \quad(n=136)$. Detailed therapeutic class-wise distribution of drugs prescribed is shown in Table 2.

91\% $(\mathrm{n}=104)$ of prescriptions prescribed with 3 large volume parenteral (LVP), followed by $6 \%(n=7)$ of prescriptions with $2 \mathrm{LVP}$ and $3 \%(\mathrm{n}=4)$ of prescriptions with 1 LVP. $\quad 61 \%(n=70)$ of prescriptions prescribed with 4 SVP, followed by $18 \%(n=21)$ of prescriptions prescribed with $3 \mathrm{SVP}, 15 \% \quad(\mathrm{n}=17)$ of prescriptions prescribed with $5 \mathrm{SVP}$ and $6 \%(\mathrm{n}=15)$ of prescriptions prescribed with 2 SVP.

In intravenous hydration Ringer lactate $36 \%(n=110)$ is the most prescribed followed by normal saline $32 \%(n=98)$, dextrose in normal saline $32 \%(\mathrm{n}=20)$. In present study $90 \%(\mathrm{n}=103)$ of patients who receiving intravenous hydration within 48 hours of symptoms. $10 \%$ of patients receiving intravenous hydration after 48 hours of symptoms onset. In our study $7 \%(\mathrm{n}=8)$ of patients receiving enteral nutrition, $3 \%(\mathrm{n}=4)$ of patients receiving parenteral nutrition.
WHO prescribing indicators like average number of drugs per prescription (8.5), percentage of drugs prescribed in generic name $61 \%$, and number of drugs prescribed from NLEM - 2015 (84\%), number of drugs prescribed from WHO essential drug list 2019 were $69 \%$ and percentage of prescriptions with injection $79.07 \%$ were shown in Table $3.6-8$

The number of prescriptions with an injection were 79.07. The overall Antibiotic encounter rate as per our study was $15 \%$. Third generation cephalosporins was the highly used antibiotics category $77 \%(\mathrm{n}=105)$ followed by penicillin's $15 \% \quad(n=21)$ and fluroquinolones $8 \% \quad(n=10)$. Most commonly prescribed antibiotics among the antibiotics was cefotaxime $(72 \%)$ followed by cefixime, ceftriaxone, piperacillin- tazobactam, ciprofloxacin $(2 \%)$. In analgesics opioids $55 \% \quad(n=98)$ are most commonly prescribed analgesics, followed by non-opioids $29 \%(\mathrm{n}=52)$ and nonsteroidal anti-inflammatory drugs (NSAID's) $16 \%(n=29)$. Most commonly prescribed drugs in analgesics are tramadol (58\%), paracetamol (33\%), diclofenac (7\%), and aceclofenac $(2 \%)$.

We calculated DDD for the drugs, which prescribed to study participants. We have calculated DDD separately for each category of drugs (obtained from the WHO ATC/DDD website). ${ }^{9}$ Based on DDD the most frequently prescribed drugs were pantoprazole, tramadol, ondansetron, octreotide followed by cefotaxime, metronidazole, paracetamol, drotaverine, sucralfate and piperacillin-tazobactam and the drugs were given ATC codes were shown in Table 4.

In present study highest ratio of PDD/DDD seen in folic acid which was 5 , and the other drug PDD/DDD ratio ranges from 0.002-0.4 (Table 4).

Table 1: Indicates etiology wise distribution according to age and gender.

\begin{tabular}{|lllllllllll|l|} 
Etiology & \multicolumn{2}{l}{ Alcoholic } & \multicolumn{2}{c}{ Gallstones } & \multicolumn{2}{c}{ Hyperlipidemic } & \multicolumn{2}{c}{ Drug induced } & \multicolumn{2}{c}{ Idiopathic } \\
Age & Male & Female & Male & Female & Male & Female & Male & Female & Male & Female \\
\hline $\mathbf{2 5 - 4 4}$ & 50 & - & 1 & 3 & 2 & 1 & - & - & 3 & - \\
\hline $\mathbf{4 5 - 6 4}$ & 24 & 1 & - & 3 & 1 & 1 & 2 & - & - & 2 \\
\hline $\mathbf{7 6 5}$ & 3 & - & 5 & 5 & 3 & 2 & - & - & 3 & - \\
\hline Percentage & 68 & & 15 & & 8 & & 2 & & 7 &
\end{tabular}

Table 2: Indicates therapeutic class of prescribed drugs.

\begin{tabular}{|llll|}
\hline S. no & Category & Frequency & Percentage \\
\hline $\mathbf{1}$ & Antiulcer agents & 153 & 21 \\
\hline $\mathbf{2}$ & Analgesics & 132 & 18 \\
\hline $\mathbf{3}$ & Antiemetics & 94 & 13 \\
\hline $\mathbf{4}$ & Somatostatin analogues & 91 & 12 \\
\hline $\mathbf{5}$ & Antibiotics & 136 & 18 \\
\hline $\mathbf{6}$ & Amebicides & 56 & 8 \\
\hline $\mathbf{7}$ & Vitamin supplements & 42 & 6 \\
\hline $\mathbf{8}$ & Anticonvulsants & 8 & 1 \\
\hline $\mathbf{9}$ & Antianxiety agents & 8 & 1 \\
\hline
\end{tabular}




\begin{tabular}{|llll|}
\hline S. no & Category & Frequency & Percentage \\
\hline $\mathbf{1 0}$ & Diuretics & 7 & 1 \\
\hline $\mathbf{1 1}$ & Insulin analogues & 7 & 1 \\
\hline $\mathbf{1 2}$ & Prokinetics & 8 & 1 \\
\hline
\end{tabular}

Table 3: Indicates WHO prescribing indicators.

\begin{tabular}{|llll|}
\hline S. no & Prescribing indicator & Values obtained & WHO standards \\
\hline $\mathbf{1}$ & Average number of drugs per patient encounter & 8.5 & $1.6-1.8$ \\
\hline $\mathbf{2}$ & Percentage of drugs prescribed by generic name & 61 & 100 \\
\hline $\mathbf{3}$ & Percentage of drugs prescribed from NLEM & 84 & 100 \\
\hline $\mathbf{5}$ & Percentage of drugs prescribed from WHO EML & 69 & 100 \\
\hline $\mathbf{6}$ & Percentage of prescriptions with injection & 79.07 & $13.4-24.1$ \\
\hline
\end{tabular}

Table 4: Indicates DDD, PDD and PDD/DDD ratio of most commonly prescribed drugs.

\begin{tabular}{|c|c|c|c|c|c|c|}
\hline S. no & Drug & ATC code & $\begin{array}{l}\text { WHO DDD } \\
\text { measure (mg) }\end{array}$ & $\begin{array}{l}\text { DDD/1000 inhabitants/day } \\
\text { (mg) }\end{array}$ & $\begin{array}{l}\text { PDD } \\
(\mathrm{mg})\end{array}$ & $\begin{array}{l}\text { PDD/ } \\
\text { DDD }\end{array}$ \\
\hline 1 & Pantoprazole & $\mathrm{A} 02 \mathrm{BC} 02$ & 40 & 100 & 80 & 2 \\
\hline 2 & Tramadol & N02AX02 & 300 & 14.8 & 100 & 0.3 \\
\hline 3 & Ondansetron & A04AA01 & 16 & 22.2 & 8 & 0.5 \\
\hline 4 & Octreotide & $\mathrm{H} 01 \mathrm{CB} 02$ & 0.7 & 12.7 & 0.2 & 0.2 \\
\hline 5 & Cefotaxime & J01DD01 & 4000 & 26.7 & 8 & 0.002 \\
\hline 6 & Metronidazole & J01XD01 & 1500 & 14.8 & 1000 & 0.6 \\
\hline 7 & Paracetamol & N02BE01 & 3000 & 11.1 & 1500 & 0.5 \\
\hline 8 & Drotaverine & A03AD02 & 100 & 8.8 & 40 & 0.4 \\
\hline 9 & Sucralfate & $\mathrm{A} 02 \mathrm{BX} 02$ & 4000 & 7.2 & 1500 & 0.3 \\
\hline 10 & $\begin{array}{l}\text { Piperacillin }+ \\
\text { tazobactam }\end{array}$ & J01CR05 & 14000 & 0.02 & 3000 & 0.6 \\
\hline 11 & Cefixime & J01DD08 & 400 & 0.1 & 400 & 1 \\
\hline 12 & Diclofenac & M02AA15 & 100 & 24.4 & 100 & 1 \\
\hline 13 & B. complex & A11EA & 1 & 46.7 & 2 & 2 \\
\hline 14 & Ranitidine & A02BA02 & 300 & 20 & 300 & 1 \\
\hline 15 & Folic acid & B03BВ01 & 0.4 & 94.4 & 2 & 5 \\
\hline
\end{tabular}

\section{DISCUSSION}

Limited data are available in general, particular in India on drug utilization on pancreatitis patients. We undertook this study in order to understand the pattern of drug use and drug related issues in pancreatitis patients.

\section{Disease condition}

Alcoholic $68 \%(n=78)$ was the most common etiology of pancreatitis followed by gallstones $15 \%(n=17)$. Study conducted by AGA reported gallstones (40-70\%) are the first most common cause of acute pancreatitis. ${ }^{10}$ Unlike in our study alcohol consumption $(68 \%)$ is the first most common and gallstones (15\%) are second most common whereas in AGA study alcoholic was the second most common $(30 \%)$. Variability in these results may be due to higher incidence alcoholic consumption in patients who visiting the study site. Incidence of gallstone pancreatitis was higher in female patients $(15 \%)$ in our study due to higher prevalence in women. ${ }^{11}$
In our study most of the patients with risk factors of alcoholic consumption $(\mathrm{n}=90)$ followed by smoking $(n=30)$, both $(n=70)$ and obesity $(n=5)$. Ammann et al stated that some patients (not all) with alcohol use disorder may have nonprogressive acute alcohol induced pancreatitis. ${ }^{12}$ Studies suggest that tobacco smoking have higher risk of pancreatitis and dose response relationship between increasing number of cigarettes use. ${ }^{13}$

In fluid resuscitation aggressive intravenous (IV) hydration with ringer lactate solution is mostly used $(n=110)$. Current guidelines recommends Ringer lactate is a preferential isotonic crystalloid replacement fluid among normal saline. Ringer lactate reduce the incidence of systemic inflammatory response syndrome as compared to normal saline. ${ }^{14}$

Most of the patients (90\%) with IV hydration within 48 hours of symptoms resume to their oral intake within a week in our study. It reflects the effectiveness of IV hydration. Our study complies with international consensus guidelines. ${ }^{15}$ 
Only $2 \%$ probiotics are prescribed. Study conducted by Gou et al concluded that probiotics do not appear effective in patients with pancreatitis. ${ }^{16}$ But in our study lower rate of probiotics are prescribed for diarrhoea associated with antibiotics therapy.

\section{Analysis of WHO prescribing indicators}

\section{Average number of drugs per prescription}

The average number of drugs per prescription was \pm 8.5 . Which is slightly higher than the WHO standards. This deviates from the WHO standards of 1.6-1.8. Minimum number of drugs per prescription was 6 and maximum number of drugs per prescription was 15 . The highest number of drugs per prescription was seen in patient with comorbidities $(10 \pm 2.23)$ patients. Also, highest number of drugs per prescription was increasing in number of hospital stay days.

Out of 115 patients, $15(13.04 \%)$ patients were taking less than five medicines per day and $100(96.96 \%)$ of patients were taking more than five medicines per day. This indicates the majority of patients in our study were having polypharmacy. Polypharmacy unfortunately is very common in India and some other countries. Considering the adverse outcomes associated with polypharmacy, including adverse drug reactions (ADR), drug-drug interactions (often very complex), increased cost of medications and/or treatment, increased risk cost of medications and/or treatment, increased risk of hospitalization, patient non-adherence with treatment (which increases with complex regimens), and various medication errors, we need to take appropriate measures for minimizing the extent of polypharmacy.

Out of 1205 drug formulations, $915(75.93 \%)$ contained only one active ingredient, while 290 (24.07\%) were FDCs. FDCs increase the risk of drug interactions and ADRs. However, the use of FDCs can improve compliance with therapy by decreasing the number of formulations to be taken. The risk/benefit ratio should be assessed before prescribing FDCs. It is not possible to increase or decrease the dose of an individual ingredient alone. However, considering that the number of FDCs in India, which is around $60 \%$ of all available formulations. ${ }^{17}$ The use of FDCs in our institution is relatively low, reflecting rational use of medicines.

\section{Number of drugs prescribed with generic name}

Out of 1205 drugs prescribed, 61\% $(n=735)$ of drugs were prescribed in generic form, $39 \%(n=400)$ were prescribed in brand name. It still falls short of the WHO recommendation of $100 \%$. However, still there is a need to encourage prescribing by generic names. Prescribing drugs by generic name led to decrease in irrational prescribing and increase in availability of essential drugs, thus ultimately promoting rational use of drugs at cheaper cost. NLEM India, promotes prescription by generic names.

\section{Number of drugs prescribed from EDL and NLEM}

We analyzed that $69 \%$ of drugs prescribed were from EDL 2019 and $84 \%$ of drugs prescribed from NLEM 2015, which is the reason why there was a very good positive outcome in patients during the treatment period.

\section{Number of prescriptions with an injection}

The number of prescriptions with an injection were $79.07 \%$, which is not appropriate with WHO recommended range of $13.4 \%-24.1 \%$. Advantage of parenteral drugs is their ability to enhance drug adherence.

\section{Number of prescriptions with antibiotic}

The overall antibiotic encounter rate as per our study was $15 \%$ that fall below the WHO recommended range of 20 $26 \%$. This reflects avoiding bacterial resistance. American college of gastroenterology (ACG) recommends use of antibiotics in extra pancreatic infections only not for prophylactic or routine use ${ }^{18}$ Our study concordance with the current recommendations of ACG guidelines.

\section{$D D D$}

DDD is the criteria used to compare utilization of drugs at international level. We used this parameter to study drug use based on dose. We calculated DDD for the drugs that were prescribed to study participants. Importance given for ten most prescribed drugs.

Based on ATC, DDD is calculated for antibiotics, analgesics (A), blood disorders and blood forming agents (B), gastrointestinal drugs and genitourinary drug (G), enzyme drugs $(\mathrm{E})$, hormonal preparation drugs $(\mathrm{H})$, and musculo skeleton drugs (M). Based on DDD pantoprazole (A02BC02), tramadol (N02AX02), ondansetron (A04AA01), octreotide (H01CB02), cefotaxime (J01DD01), metronidazole (J01XD01), paracetamol (N02BE01), drotaverine (A03AD02), sucralfate (A02BX02), and piperacillin-tazobactam (J01CR05).

Pantoprazole (A02BX02), sucralfate (A02BX02) comes under gastrointestinal drugs is being prescribed for gastric hypersecretion and gastric ulcer. Cefixime (J01DD08), cefotaxime (J01DD01), metronidazole (J01XD01) and piperacillin-tazobactam (J01CR05) comes under antibiotics is being prescribed for infected necrosis and extra pancreatic infections. Metronidazole penetrate pancreatic necrosis tissue and delay or avoid surgical intervention and to decrease morbidity and mortality. ${ }^{18}$ Tramadol (N02AX02) an opioid analgesic comes under analgesics mainly for moderate to severe pain and paracetamol (N02B01), diclofenac (M02AA15) are NSAID's comes under analgesics mainly for mild to moderate pain. Basurto et al study suggested opioids are the safe and effective in pancreatitis. ${ }^{19}$ Compared to 
other opioids tramadol was acting at both the opioid receptors as well as serotonin-norepinephrine reuptake inhibition and lesser abuse potential and cost effective in management of pancreatic pain. ${ }^{20}$

Octreotide (H01CB02) comes under hormonal preparations is prescribed for suppressing pancreatic secretions. It reduce mortality in patients with acute severe pancreatitis. $^{21}$

\section{PDD/DDD ratio}

Cefixime, diclofenac, ranitidine had PDD/DDD ratios equal to 1. Most of the drugs had the PDD/DDD ratio below 1 and the drugs pantoprazole, paracetamol, thiamine, B-complex and folic acid (highest PDD/DDD ratio) had their ratios above 1 .

Drugs with PDD/DDD ratio lesser or greater than 1 are either under or over utilized. Often PDD can vary due to factors like illness treated, and national therapeutic procedures. For example, PDDs are often lower in Asian than in Caucasian populations. It does not mean there is underutilization of drug. Also WHO ATC/DDD for management of moderate intensity hence the WHO encourages countries to have their own DDD list based on local data. ${ }^{22}$

\section{Limitations}

Sample size of our study is small and study period is 6 months. We could not compare the effectiveness and adverse effect profile of various analgesics. At the same time our study has numerous strengths. Ours was the first study done in this area regarding utilization of drugs in conservative management of pancreatitis. We have used various drug utilization metrics like ATC/DDD classification, PDD, PDD/DDD ratios to present our data in a scientific manner to enable comparison with other studies.

\section{CONCLUSION}

This study provides a baseline data regarding the prescribing pattern of drugs used in conservative management of patients with pancreatitis. Drug utilization studies are need of the hour for rational prescription of drugs.

Our findings shown that majority of patients were on polypharmacy. Polypharmacy is reduced by reviewing previous medication history, monitor for adverse drug withdrawal events, assess for drug-drug interactions and also use tools such as beers criteria, START/STOPP criteria, deprescribing, medication appropriateness index and ARMOR protocols. Pharmacists' especially clinical pharmacist, doctor of pharmacy (Pharm D) who intervene drug therapy and drug related problems in clinical scenario play a vital role in reducing polypharmacy. The prescription of drugs in brand name could be changed to generic name. The use of drugs from national and WHO essential medicine list needs to be increased in order to have more rational drug prescribing.

There is always scope for improvement of rational prescribing by introducing appropriate educational interventions, which may be considered as an effort to improve quality of health care. This study provided a scope for further research in this area.

\section{ACKNOWLEDGEMENTS}

Authors would like to thank to Rajah Muthiah Medical College and Hospital for allowing to conduct this study and they would also like to thank parents and friends.

Funding: No funding sources Conflict of interest: None declared

Ethical approval: The study was approved by the Institutional Ethics Committee

\section{REFERENCES}

1. World Health Organization. Introduction to drug utilization research. 2003. Available at: https://apps.who.int/iris/handle/10665/42627. Accessed on 17 July 2021.

2. Dinesh Kumar Meena M. Jayanthi-Drug Utilization research: a review Int $\mathbf{J}$ of Basic Clinical Pharmcol. 2019;8(2):354-61.

3. World Health Organization. DDD indicators. Available at: https://www.who.int/medicines/regulat ion/medicines-safety/toolkit_indicators/en/index 1 .html. Accessed on 17 July 2021.

4. World Health Organization. Action programme on Essential Drugs and Vaccines. How to investigate drug use in facilities. Selected drug use indicators. WHO. 1993. Available at: https://apps.who.int/ iris/handle/10665/60519. Accessed on 17 June 2021.

5. Capellà D. Descriptive Tools and Analysis. In Drug Utilization Studies: Methods and Uses. Edited by Dukes MNG. WHO Regional Publications, European Series, No. 45. 1992;55-78.

6. World health organization. Prescribing indicators. Available at: https://apps.who.int/medicinedocs/ en/d/Js2289e/3.1.html. Accessed on 17 June 2021.

7. National List of Essential Medicines-2015. Central Drugs Standard. Available at: https://www.cdsco.nic. in/National\%20List\%20of\%20Essential\%20Medicin e-\%20final \%20copy.pdf. Accessed on 17 July 2021.

8. WHO Essential Medicines. 2019. Available at: https://apps.who.int/iris/bitstream/handle/10665/3257 71/WHO-MVP-EMP-IAU-2019.06-eng.pdf. Accessed on 17 July 2021.

9. ATC/DDD Index 2012. WHO Collaborating Centre for Drug statistics Methodology Norwegian Institute of Public Health. 2012. Available at: https://www.whocc.no/. Accessed on 17 July 2021.

10. Forsmark CE, Baillie J, AGA Institute Clinical Practice and Economics Committee, AGA Institute 
Governing Board. AGA Institute technical review on acute pancreatitis. Gastroenterology. 2007;132(5):2022-44.

11. Yang AL, Vadhavkar S, Singh G, Omary MB. Epidemiology of alcohol-related liver and pancreatic disease in the United States. Arch Intern Med. 2008;168(6):649-56.

12. Ammann RW, Heitz PU, Klöppel G. Course of alcoholic chronic pancreatitis: a prospective clinicomorphological long-term study. Gastroenterology. 1996;111(1):224-31.

13. Aune D, Mahamat-Saleh Y, Norat T, Riboli E. Tobacco smoking and the risk of pancreatitis: A systematic review and meta-analysis of prospective studies. Pancreatology. 2019;19(8):1009-22.

14. Wu BU, Hwang JQ, Gardner TH, Repas K, Delee R, Yu S, Smith B, Banks PA, Conwell DL. Lactated Ringer's solution reduces systemic inflammation compared with saline in patients with acute pancreatitis. Clin Gastroenterol Hepatol 2011;9(8):710-7.

15. Mirtallo JM, Forbes A, McClave SA, Jensen GL, Waitzberg DL, Davies AR; International Consensus Guideline Committee Pancreatitis Task Force. International consensus guidelines for nutrition therapy in pancreatitis. J Parenter Enteral Nutr. 2012;36(3):284-91.

16. Gou S, Yang Z, Liu T, Wu H, Wang C. Use of probiotics in the treatment of severe acute pancreatitis: a systematic review and meta-analysis of randomized controlled trials. Crit Care. 2014;18(2):57.

17. Arshad M, Fayazuddin M, Mudium R, Chavan VR. Drug utilization study among geriatric patients in a tertiary care teaching hospital in south India. Asian J Pharm Clin Res. 2019;12(10):245-8.

18. Tenner S, Baillie J, DeWitt J, Vege SS, American College of Gastroenterology. American College of Gastroenterology guideline: management of acute pancreatitis. Am J Gastroenterol. 2013;108(9):140015.

19. Basurto Ona X, Rigau Comas D, Urrútia G. Opioids for acute pancreatitis pain. Cochrane Database Syst Rev. 2013;(7):CD009179.

20. Wilder-Smith CH, Hill L, Osler W, O'Keefe S. Effect of tramadol and morphine on pain and gastrointestinal motor function in patients with chronic pancreatitis. Dig Dis Sci. 1999;44:1107-16.

21. Moggia E, Koti R, Belgaumkar AP, Fazio F, Pereira SP, Davidson BR, Gurusamy KS. Pharmacological interventions for acute pancreatitis. Cochrane Database Syst Rev. 2017;4(4):CD011384.

22. WHO International Working Group of Drug Statistics Mehtodology. Introduction to drug utilization research. Geneva: WHO collaborating Centre for Drug Utilization Research and Clinical Pharmacology. 2003. Available at: https://apps.who.int/iris/ handle/10665/42627. Accessed on 17 July 2021.

Cite this article as: Mohanraj $\mathrm{P}$, Abinaya S, Shalini K, Gopalakrishnan G, Ravichandran K. Study on drug utilization pattern in conservative management of patients with pancreatitis in a tertiary care teaching hospital. Int J Basic Clin Pharmacol 2021;10:1286-92. 